University of Nebraska - Lincoln

DigitalCommons@University of Nebraska - Lincoln

March 1994

\title{
Exchange Coupling and Energy Product in Random Two- Phase Aligned Magnets
}

\author{
Ralph Skomski \\ University of Nebraska-Lincoln, rskomski2@unl.edu \\ J.M.D. Coey \\ Trinity College, Dublin, Ireland
}

Follow this and additional works at: https://digitalcommons.unl.edu/physicsskomski

Part of the Physics Commons

Skomski, Ralph and Coey, J.M.D., "Exchange Coupling and Energy Product in Random Two- Phase Aligned Magnets" (1994). Ralph Skomski Publications. 4.

https://digitalcommons.unl.edu/physicsskomski/4

This Article is brought to you for free and open access by the Research Papers in Physics and Astronomy at DigitalCommons@University of Nebraska - Lincoln. It has been accepted for inclusion in Ralph Skomski Publications by an authorized administrator of DigitalCommons@University of Nebraska - Lincoln. 


\title{
Exchange Coupling and Energy Product in Random Two- Phase Aligned Magnets
}

\author{
R. Skomski and J. M. D. Coey \\ Department of Pure and Applied Physics, Trinity College, Dublin 2, Ireland
}

\begin{abstract}
Size effects in two-phase Boolean magnets composed of an allgned hard phase and a soft phase with high magnetization are investigated. In 'overcritical' magnets, where the size of the soft regions is larger than the Blochwall width $\delta_{h}$ of the hard phase, the energy product is reduced compared to the corresponding 'undercritical' magnets with small soft regions, where theoretical energy products as high as 1 $M \mathrm{~J} / \mathrm{m}^{3}$ have been predicted. Nevertheless, the theoretical energy product only gradually drops with increasing size of the soft regions, and it may still exceed the theoretical energy product 516 $\mathrm{kJ} / \mathrm{m}^{3}$ of $\mathrm{Nd}_{2} \mathrm{Fe}_{14} \mathrm{~B}$.
\end{abstract}

\section{INTRODUCTION}

The energy product $(\mathrm{BH})_{\max }$, which gives the maximum magnetostatic energy available from a magnet, is a key figure of merit for permanent magnetic materials. The energy product increases with the coercivity but never exceeds its theoretical value $\mu_{0} \mathrm{M}_{0}{ }^{2} / 4$. The theoretical energy product of bcc iron is $\mu_{\mathrm{o}} \mathrm{M}_{\mathrm{o}}^{2} / 4=920 \mathrm{~kJ} / \mathrm{m}^{3}$, but in fact pure iron exhibits nearly no coercivity so the large theoretical energy product is far from being realized in practice. In modern permanent magnetic alloys such as $\mathrm{Nd}_{2} \mathrm{Fe}_{14} \mathrm{~B}$ [1] and $\mathrm{Sm}_{2} \mathrm{Fe}_{17} \mathrm{~N}_{3}$ [2], the saturation magnetization does not reach the high value $\mu_{0} M_{0}=2.15 \mathrm{~T}$ of iron but the high uniaxial anisotropy makes them useful permanent magnets. At present, energy products as high as $405 \mathrm{~kJ} / \mathrm{m}^{3}$ are obtained in laboratory-scale $\mathrm{Nd}_{2} \mathrm{Fe}_{14} \mathrm{~B}$ magnets [1].

The outlook for discovering new ternary phases with significantly higher magnetization than those available at present is poor [3], but as it has been shown recently [4], exchange coupling in nanostructured aligned two phase magnets consisting of alloys available at present yields theoretical energy products exceeding that of $\mathrm{Nd}_{2} \mathrm{Fe}_{14} \mathrm{~B}$ ('giant energy product'). The nucleation field, which determines the energy-product in nucleation-controlled magnets, is calculated from the magnetic free energy integral [5] - [8]

$$
\begin{aligned}
F=\int & {\left[A(r)\left(\frac{\nabla M_{i}}{M_{o}(r)}\right)^{2}-K_{1}(r)\left(\frac{M_{i} n_{i}}{M_{o}(r)}\right)^{2}-\mu_{o} M_{i} H_{i}\right] d r } \\
& -\frac{1}{2} \sum_{i, j=1}^{3} \int \mathbb{K}_{i j}\left(\mathbf{r}-\mathbf{r}^{\prime}\right) M_{i}(\mathbf{r}) M_{j}\left(r^{\prime}\right) d \mathbf{r} d r^{\prime}
\end{aligned}
$$

where $\mathrm{A}(\mathbf{r})$ is the exchange stiffness, the $\mathrm{M}_{\mathrm{i}}(\mathbf{r})$ with $|\mathbf{M}(\mathbf{r})|=$ $M_{0}$ denote the components of the local magnetization, and $\mathrm{K}_{1}(\mathbf{r})$ the first anisotropy constant. $\mathrm{H}_{\mathrm{i}}=\mathrm{e}_{\mathrm{zi}} \mathrm{H}_{\mathrm{z}}$ denotes the external magnetic field, $n_{i}$ is the unit vector of the easy-axis direction, and the nonlocal kernel $\mathbb{K}_{\mathrm{ij}}\left(\mathbf{r}-\mathbf{r}^{\prime}\right)$ describes the magnetostatic dipole interaction.

The basic assumptions made in [4] are:

(i) The size of the soft regions is smaller than or comparable to the domain-wall width of the hard phase. This leads to an exchange coupling between hard and soft regions which is comparable to the well-known remanence enhancement in isotropic two-phase magnets (cf. [4] , [9]).

(ii) The hard regions are aligned $\left(n_{i}=e_{z i}\right)$ so the exchange coupling leads to an effective increase in remanence magnetization and energy product.

(iii) The first order anisotropy constant $K_{h}$ of the hard phase is much larger than magnetostatic energy density of the soft phase.

The upper limit of the theoretical energy product is [4]

$$
(\mathrm{BH})_{\max }=\frac{1}{4} \mu_{\mathrm{o}}\left\langle\mathrm{M}_{\mathrm{o}}\right\rangle^{2}\left(1-\frac{\mu_{\mathrm{o}}\left(\mathrm{M}_{\mathrm{s}}-\mathrm{M}_{\mathrm{h}}\right) \mathrm{M}_{\mathrm{s}}}{2 \mathrm{~K}_{\mathrm{h}}}\right)
$$

where $M_{s}$ and $M_{h}$ denote the magnetizations of the soft and hard phases, respectively [4], and $\left\langle\mathrm{M}_{\mathrm{o}}\right\rangle=\mathrm{f}_{\mathrm{s}} \mathrm{M}_{\mathrm{s}}+\mathrm{f}_{\mathrm{h}} \mathrm{M}_{\mathrm{h}}$ the average magnetization of the magnet. For instance, Eq. (2) predicts an energy product as high as $1090 \mathrm{~kJ} / \mathrm{m}^{3}$ for the system $\mathrm{Sm}_{2} \mathrm{Fe}_{17} / \mathrm{Fe}_{65} \mathrm{Co}_{35}$, with a volume fraction of the hard phase $\mathrm{f}_{\mathrm{h}}$ of only $11 \mathrm{vol} . \%$.

However, the production of magnets structured on a scale smaller than the Bloch-wall width $\delta_{\mathrm{h}}$ of the hard phase is a difficult problem, and in practice it might be suitable to resort to magnets where the soft regions are larger than $\delta_{h}$. It is well known that there is no closed general solution for the corresponding micromagnetic problem [5] - [8], and only for a few configurations, such as homogeneous ellipsoids, the nucleation problem can be solved analytically [7]. Here we investigate the effect of exchange-stiffness and anisotropy inhomogenities, and random magnetic stray fields to predict the behaviour of magnets structured on a overcritical scale.

\section{RESULTS AND DISCUSSION}

\section{Random stray fields}

From the theoretical point of view, the magnetostatic interaction is the most difficult in (1) to deal with. This is due to the non-local kernel 


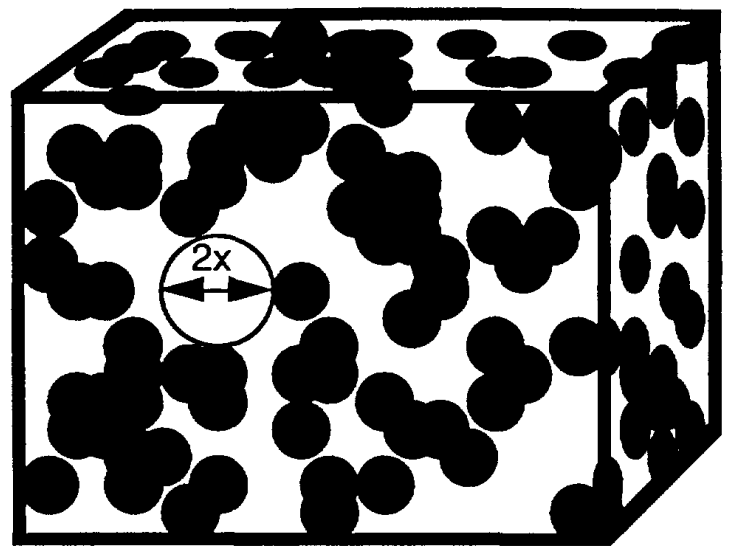

Fig. 1. Boolean model describing the structure of disordered twophase magnets. The position of the spheres (hard regions) is random, but many geometrical properties, such as the typical distance $x$ to the next sphere, are given analytically [15].

$$
\mathbb{K}_{i j}(\mathbf{r})=\frac{\mu_{0}}{4 \pi} \frac{3 r_{i} r_{j}-\delta_{i j} r^{2}}{r^{5}}
$$

which describes the long-range dipole interaction between different magnetic regions. A simple but nevertheless nontrivial approximation is to replace $\mathrm{H}_{\mathrm{Z}}$ in (1) by $\mathrm{H}_{\mathrm{Z}}-\mathrm{D}\left\langle\mathrm{M}_{\mathrm{o}}\right\rangle$, where $\mathrm{D}$ is the macroscopic demagnetization factor of the ellipsoid.

In disordered aligned two-phase magnets (cf. Fig. 1) there are random stray fields which are incompatible with the assumption of a homogeneous demagnetizing field. These stray-field inhomogenities are harmful to coercivity if they cover a volume larger than about $\delta h^{3}$. Smaller stray-field inhomogenities are ineffective: exchange coupling prevents regions smaller than $\delta_{h}$ from being switched even if they are subject to a strong demagnetizing field.

The local stray fields are given by

$$
\mu_{0} H_{i} L^{L}(\mathbf{r})=\int \sum_{j=1}^{3} \mathbb{K}_{i j}\left(\mathbf{r}-\mathbf{r}^{\prime}\right) M_{j}\left(\mathbf{r}^{\prime}\right) d \mathbf{r}^{\prime}
$$

where the integration includes all points inside a sufficiently large sphere. Note that the angular integration $\sin \theta d \theta$ yields zero for all contributions spherical around $r$. In particular, $\mathrm{H}_{\mathrm{i}} \mathrm{L}(\mathbf{r})=\left\langle\mathrm{H}_{\mathrm{i}} \mathrm{L}(\mathbf{r})\right\rangle=0$ for $\mathrm{M}_{\mathrm{i}}(\mathbf{r})=\left\langle\mathrm{M}_{\mathrm{i}}(\mathbf{r})\right\rangle$, where $\langle\ldots\rangle$ denotes the volume average.

Situation changes if the variation of $\mathrm{H}_{\mathrm{i}} \mathrm{L}(\mathbf{r})$ is considered. To calculate $\left\langle\left[\mathrm{H}_{\mathrm{i}} \mathrm{L}(\mathbf{r})\right]^{2}\right\rangle=\left(\delta \mathrm{H}_{\|}\right)^{2}+2\left(\delta \mathrm{H}_{\perp}\right)^{2}$, we have to know the correlation function $\left\langle\mathrm{M}_{\mathrm{o}}(\mathbf{r}) \mathrm{M}_{\mathrm{o}}\left(\mathbf{r}^{\prime}\right)\right\rangle$ which will vary from sample to sample. A reasonable, since largely uncorrelated, ansatz is

$$
\left\langle M_{\mathrm{o}}(\mathbf{r}) \mathrm{M}_{\mathrm{o}}\left(\mathbf{r}^{\prime}\right)\right\rangle=\left\langle\mathrm{M}_{\mathrm{o}}\right\rangle^{2}+\mathrm{V}_{\mathrm{o}} \delta\left(\mathbf{r}-\mathbf{r}^{\prime}\right)\left[\left\langle\mathrm{M}_{\mathrm{o}}{ }^{2}\right\rangle-\left\langle\mathrm{M}_{\mathrm{o}}\right\rangle^{2}\right]
$$

describing inhomogenities whose size (correlation length) is of order $\mathrm{V}_{0} 1 / 3$. Calculation yields

$$
\left(\delta \mathrm{H}_{\|}\right)^{2}=\frac{4}{15}\left[\left\langle\mathrm{M}_{\mathrm{o}}^{2}\right\rangle-\left\langle\mathrm{M}_{\mathrm{o}}\right\rangle^{2}\right]
$$

and

$$
\left(\delta \mathrm{H}_{\perp}\right)^{2}=\frac{1}{5}\left[\left\langle\mathrm{M}_{\mathrm{o}}^{2}\right\rangle-\left\langle\mathrm{M}_{\mathrm{o}}\right\rangle^{2}\right]
$$

For the $\mathrm{Sm}_{2} \mathrm{Fe}_{17} / \mathrm{Fe}_{65} \mathrm{Co}_{35}$ system we predict $\mu_{0} \delta \mathrm{H}_{\|}=$ $0.07 \mathrm{~T}$. This means that random stray fields in typical aligned two-phase magnets hardly exceed a few tenth of a Tesla, and a small compensating increase of the volume fraction $\mathrm{f}_{\mathrm{h}}$ of the hard phase will be sufficient to re-establish the necessary coercivity.

\section{Exchange stiffness and Inhomogeneous Anisotropy}

We now neglect random stray fields in (1) and expand the free energy with respect to the small transverse components $m_{x}=M_{x} / M_{0} \ll 1$ and $m_{y}=M_{y} / M_{0} \ll 1$ :

$$
\begin{array}{r}
F=F_{o}+\int\left[A(r)\left(\nabla m_{i}\right)^{2}+K_{1}(r) m_{i}{ }^{2}-\right. \\
\left.-\frac{1}{2} \mu_{o} m_{i}{ }^{2} M_{o} H_{z}\right] d r
\end{array}
$$

Next we have to calculate the free-energy minimum and to look whether the equilibrium is stable or not. To minimize $F$ $=\int \eta \mathrm{dr}$ we use the formula

$$
\frac{\delta F}{\delta m_{i}}=-\nabla\left(\frac{\partial \eta}{\partial \nabla m_{i}}\right)+\frac{\partial \eta}{\partial m_{i}}=0
$$

where $\delta \mathrm{F} / \delta \mathrm{m}_{\mathrm{i}}(\mathbf{r})$ denotes the functional derivative (see e.g. [10]) and obtain the eigenvalue problem

$$
-\nabla\left[A(r) \nabla m_{i}\right]+K_{1}(r) m_{i}=\frac{1}{2} \mu_{0} M_{o} H_{N} m_{i}
$$

with the nucleation field $\mathrm{H}_{\mathrm{N}}$. Note that the two components $\mathrm{m}_{\mathrm{i}}$ are decoupled in demagnetizing-field approximation.

The $\nabla A(\mathbf{r})$-contribution in $(10)$ is due the fact that the magnetization in magnets with large exchange stiffness tends to be more homogeneous than in those with a lower exchange stiffness. However, $A$ is of order $10^{-11} \mathrm{~J} / \mathrm{m}$ in both phases so the $\nabla A(r)$-term, though included in [4], does not change the qualitative behaviour of the system. In particular, it does not affect the energy product in overcritical two-phase magnets.

The $K_{1}(r)$ term in (10) describes the effect of a soft phase, where $\mathrm{K}_{1}(r)=\mathrm{K}_{\mathrm{s}} \approx 0$, on the nucleation field $\mathrm{H}_{\mathrm{N}}=$ $\mathrm{H}_{\mathrm{Z}}$. To calculate the nucleation field, we use the similiarity between Eq. (10) with $\nabla A(r)=0$ and Schrödinger's equation for a particle moving in a three-dimensional potential $U(r)=$ $\mathrm{K}_{1}$ (r) (cf. [4]). Up to second order perturbation theory

$H_{N}=\frac{2\left\langle K_{1}(r)\right\rangle}{\mu_{0} M_{o}}-\frac{2}{\mu_{0} M_{o} A V} \Sigma_{k}\left(k^{-2} \int_{v} e^{i k r}\left\langle K_{1}(r) K_{1}(0)\right\rangle d r\right)$

where the $\mathrm{k}$ summation includes all plane waves exp(ikr). It can be shown that this assumption is reasonable for macros- 
copically large magnets, where modes such as curling are curling are negligible (see e.g. [3] and [5] - [8]).

The linear term in (11) corresponds to the introduction of a volume-averaged anistropy constant $\mathrm{K}_{\mathrm{eff}}(\boldsymbol{r})=\left\langle\mathrm{K}_{1}(\mathbf{r})\right\rangle$ and reproduces the energy product in (2). As can be shown by using the quantum-mechanical analogy, the quadratic term in (11) always reduces $H_{N}$, so this equation may serve as a simple illustration of Brown's paradox. As opposed to the first-order correcture, which does not depend explicilly on the geometrical form of the soft and hard regions, the second order contribution involves the correlation function $\left\langle\mathrm{K}_{1}(\mathbf{r}) \mathrm{K}_{1}(0)\right\rangle$. This correlation function strongly depends on the real structure of the magnet, and it is not possible any longer to obtain closed expressions such as (2).

Equation (11) gives the first two terms of a perturbation series. The small parameter of this expansion is $s 2 / \delta h^{2}$, where $s$ is the correlation length of the inhomogenities, i.e. roughly speaking the size of the hard and soft regions. For small s a 'plateau' behaviour [4] is obtained, whereas the series (11) breaks down for large correlation lengths (for other approaches see e.g. [11] - [14]).

Compared to random stray fields and exchange-stiffness inhomogenities, large soft regions are much more harmful to coercivity and energy product. To discuss this effect we use a Boolean model [15] where overlapping randomly distributed spheres with radius $\mathrm{R}$ serve to model the hard regions Fig. 1 . For this model the probability $\mathrm{p}(\mathrm{x}>\mathrm{L})$ that the distance $\mathrm{x}$ between a typical soft-phase point and the closest hard-phase point is larger than $L$ [15]

$$
\mathrm{p}(\mathrm{x}>\mathrm{L})=\left(1-\mathrm{f}_{\mathrm{h}}\right)^{(1+\mathrm{L} / \mathrm{R})^{3}-1}
$$

To remain in the plateau region we have to choose $L=\delta \mathrm{h} / 2=$ $1.5 \mathrm{~nm}$. On the other hand, the size of the hard regions cannot be smaller than about $1 \mathrm{~nm}$ [4], so we obtain with the typical value $\mathrm{f}_{\mathrm{h}}=11 \%$ the probability $\mathrm{p}(\mathrm{x}>1.5 \mathrm{~nm})=0.06 \%$. This means that less than one out of 1000 soft-phase atoms are in a too-large region, which makes the production of macroscop-ically large magnets with totally random distribution of the hard phase unrealistic. However, a small increase of the fraction of the hard phase strongly improves the prospects for making a reliable magnet: taking $\mathrm{f}_{\mathrm{h}}=20 \%$ instead of $f_{h}=10 \%$ reduces the energy product of the $\mathrm{Sm}_{2} \mathrm{Fe}_{17} / \mathrm{Fe}$ system by only $6 \%$, while $\mathrm{p}(\mathrm{x}>1.5 \mathrm{~nm})=7.8$ $10-5 \%$. A similiar effect is achieved by leaving the plateau region, which must be compensated by increasing $f h$, and structuring the magnet on a somewhat larger scale. Note that these difficulties do not occur if the hard regions a more regularly dispersed.

\section{CONCLUSIONS}

Disordered two-phase magnets with a common $\mathrm{c}$ axis throughout the hard regions structured on an 'overcritical' scale are subject to random stray fields which reduce the coercivity by a few tenth of a Tesla. More harmful to coercivity are fluctuations of the size of the soft regions, which occur in particular in totally random structures. Using a Boolean model we have calculated nucleation probabilities which show that increasing the amount of the hard phase strongly improves the skeleton performance of the hard phase in 'overcritical' magnets. This stabilization is paid by a slight decrease in saturation magnetization, so the ultimate energy product $1090 \mathrm{~kJ} / \mathrm{m}^{3}$ can be approached but not reaches in 'overcritical' magnets.

\section{ACKNOWLEGDMENTS}

This work forms part of the 'Concerted European Action on Magnets'. It was supported by the BRITE/EURAM Programme of the European Commission.

\section{REFERENCES}

[1] M. Sagawa, S. Hirosawa, H. Yamamoto, S. Fujimura, and Y. Matsuura, Jpn. J. Appl. Phys., vol. 26, p. $785,1987$.

[2] J. M. D. Coey and H. Sun, J.Magn.Magn.Mater., vol. 87, p. L251, 1990; J. M. D. Coey, Physica Scripta, vol. T39, p. $21,1991$.

[3] J. M. D. Coey and R. Skomski, Physica Scripta, in press, 1993.

[4] R. Skomski and J. M. D. Coey, IEEE Trans. Mag., in press, 1993; R. Skomski and J. M. D. Coey, Phys. Rev. B., in press, 1993.

[5] W. F. Brown, Phys. Rev., vol. 58, p. 736, 1940.

[6] W. F. Brown, Rev. Mod. Phys., vol. 17, p. 15, 1945.

[7] A. Aharoni, Rev. Mod. Phys., vol. 34, p. 227, 1962.

[8] W. F. Brown, Micromagnetics, Interscience, New York 1963.

[9] R. Coehoorn, D. B. de Mooij. J. P. W. Duchateau, and K. H. J. Buschow, J. Physique., vol. 49 , p. C8-669, 1991.

[10] E. Fick, Einführung in die Grundlagen der Quantenmechanik, Geest\&Portig, Leipzig 1981.

[11] H. Kronmüller, phys. stat. sol. (b), vol. 144, p. 385, 1987.

[12] S. Nieber and H. Kronmüller, phys. stat. sol. (b), vol. 153 , p. $367,1989$.

[13] R. Skomski, phys. stat. sol. (b)., vol. 174, p. K77, 1992.

[14] Jifan Hu and H. Kronmüller, phys. stat. sol. (b)., vol. 172, p. $673,1992$.

[15] D. Stoyan and J. Mecke, Stochastische Geometrie, Akademie-Verlag, Berlin 1983. 